\title{
The Use of Probiotics in the Prevention of C. difficile Infection in Admitted Patients Who were Given Broad-Spectrum Antibiotics
}

\author{
Cynthia Uche ${ }^{1 *}$, Mary Wallace ${ }^{2}$, Tatiyana Makarevich ${ }^{2}$, Laural Farabaugh ${ }^{2}$ and Yuliya Klopouh ${ }^{3}$ \\ ${ }^{1}$ Department of Pharmacy Practice and Science, University of Maryland School of Pharmacy, USA \\ ${ }^{2}$ Infection Prevention and Control, Northwest Hospital, USA \\ ${ }^{3}$ Pharmacy Department, Northwest Hospital, USA \\ *Corresponding Author: Cynthia Uche, Department of Pharmacy Practice and Science, University of Maryland School of Pharmacy, USA
}

Received: November 13, 2019; Published: November 14, 2019

DOI: 10.31080 /ASPS.2019.03.0437

\begin{abstract}
Probiotics are microorganisms that are usually administered as a supplement for the maintenance of normal gastrointestinal, GI, bacterial flora. For patients with a compromised GI tract, due to antibiotic use, the administration of probiotics is thought to help reduce the development of opportunistic infections such as $C$. difficile. In this study, the Lactobacillus species was the probiotic of choice. Meta-analysis studies showed that probiotics may be effective in reducing the morbidity of $C$. difficile infection. The present study highlights the protocol used, in an inpatient setting, to help reduce the rates of C. difficile infection by prophylactic administration of probiotics to patients receiving six broad-spectrum antibiotics. The two probiotics used were Floranex ${ }^{\mathrm{TM}}$ and Culturelle $^{\circledR}$. This initiative also involved the implementation of an environmental cleaning protocol, the Clean Collaborative. A two-step process for probiotics implementation was done by pharmacists. A trend towards lower $C$. difficile infection rates per 10,000 patient days was observed at the conclusion of the program.
\end{abstract}

Keywords: Probiotics; Clostridium difficile; Gastrointestinal Health; Lactobacillus; Broad Spectrum Antibiotics

\section{Abbreviations}

CDI: C. difficile Infection; HO-CDI: Hospital-Onset Clostridium difficile Infections; NHSN: National Healthcare Safety Network; NWH: Northwest Hospital; and CYTD: Current Year to Date.

\section{Introduction}

The use of broad-spectrum antibiotics for empiric treatment, in an inpatient hospital setting, can lead to a significant reduction in the normal bacterial flora of the intestines, resulting in opportunistic infections [1]. Clostridium difficile, C. difficile, is a common opportunistic organism, which leads to the infection of the intestines. C. difficile infections have caused 14,000 deaths in the United States, annually. Studies have shown that $C$. difficile has been associated with increased hospital stay, cost of care, morbidity, and mortality [2]. The financial burden of $C$. difficile infections on the U.S. national healthcare system has been estimated to be $\$ 1.3$ billion dollars annually [2]. During the implementation of this protocol, the National Health Safety Network, NHSN, definition of $C$. difficile infection (CDI), which requires laboratory identification of the bacterium was used [3]. The number of laboratory identified hospital-onset Clostridium difficile infections (HO-CDI) in Maryland was $20 \%$ higher than the national baseline [3]. Prophylactic treatment with probiotics along with good environmental cleaning practices may help reduce the chance of developing HO-CDI during a course of antibiotics [1,2].

Probiotics are microorganisms that are usually administered as a supplement for the maintenance of normal gastrointestinal, GI, bacterial flora [4]. For patients with a compromised GI tract, es- 
The Use of Probiotics in the Prevention of $C$. difficile Infection in Admitted Patients Who were Given Broad-Spectrum Antibiotics

pecially due to antibiotic use, the administration of probiotics is thought to help reduce the development of opportunistic infections such as $C$. difficile $[4,5]$. In this study, the Lactobacillus species, a gram positive, facultative anaerobic rod shaped bacterium, was the probiotic of choice. Lactobacillus species are one of the most numerous in the GI tract. This organism is one of the most numerous of the normal GI flora, and had the most promising results [5-7]. Meta-analysis studies also showed that probiotics may be effective in reducing morbidity of CDI [5-7]. The present study highlights the protocol used, in an inpatient setting, to help reduce the rates of CDI by prophylactic administration of probiotics to patients receiving six broad-spectrum antibiotics. The two probiotics used were Floranex ${ }^{\mathrm{TM}}$ and Culturelle ${ }^{\circledR}$. Floranex ${ }^{\mathrm{TM}}$ Granules contain Lactobacillus acidophilus and Lactobacillus bulgaricus 100 million live cells per $1 \mathrm{~g}$ packet. Culturelle ${ }^{\circledR}$ contains 10 billion live active cultures of Lactobacillus GG (Lactobacillus rhamnosus). Lactobacillus (rhamnosus) GG, acidophilus and bulgaricus have been shown to improve antibiotic associated diarrhea, especially in combination [8]. This initiative also involved the implementation of an environmental cleaning protocol, the Clean Collaborative, to inpatient rooms and other areas susceptible to exposure to $C$. difficile.

Initial surveillance

This probiotics protocol implementation program was conducted at Northwest Hospital (NWH), a 258 bed hospital located in Randallstown, Maryland, from May 2016-May 2017. The program included pharmacists and pharmacy students. No additional staff was used for the probiotic administration portion of the project. After monitoring of HO-CDI cases through daily surveillance using C. difficile LabID National Healthcare safety Network (NHSN) criteria, defect analysis was performed on HO-CDI cases. Defect analysis showed that nearly all patients (approximately 90\%) were on antibiotics.

\section{Methodology}

\section{Probiotics administration}

The study included all admitted patients, in all units except the sub-acute unit, who were receiving any of the six broad spectrum antibiotics being studied: Zosyn, ciprofloxacin, levofloxacin, ceftriaxone, cefepime, and clindamycin. These six antibiotics were identified as the most frequently used for empiric treatment of systemic infection. They are also associated with an increased risk of $C$. difficile infection. Patients excluded from the protocol were those who were on chemotherapy/radiotherapy (i.e. immunocompromised), and those with white blood cell count (WBC)<4000 mcg/L. Lastly,
If any of the 6 antibiotics were administered as a one time dose from the emergency department therefore, not in the control of the pharmacists, those patients were excluded as well.

\section{Administration protocol}

Floranex ${ }^{\mathrm{TM}}$ was only administered to patients who could tolerate medication and food by mouth. Patients who were on any of the six antibiotics and receiving nutrition and medication through nasogastric/gastric tube were given Culturelle ${ }^{\circledR}$. Culturelle ${ }^{\circledR}$ was chosen after surveillance information from Mt. Washington Pediatric Hospital showed that Culturelle ${ }^{\circledR}$ was the best probiotic for administration in small feeding tubes. The probiotics were given to a patient within 24-hours of administration of any of 6 antibiotics.

\section{Floranex ${ }^{\mathrm{TM}}$ administration}

Probiotics were ordered as Floranex ${ }^{\mathrm{TM}}$ tablets ( 4 tablets given 3-4 times daily) or granule packets (1 packet given 3-4 times daily). The packets were then loaded in AccuDose $\mathrm{e}^{\mathrm{TM}}$ machines. Neither the packets nor the tablets need refrigeration. 1 packet of Floranex ${ }^{\mathrm{TM}}$ granules was added to or taken with water, fruit juice, milk, food, or cereal and administered to the patient.

\section{Culturelle ${ }^{\circledR}$ administration}

After flushing the feeding tube with $15-30 \mathrm{~mL}$ of water, the powder from one capsule was diluted in $10-15 \mathrm{~mL}$ of water. Prior to drawing up into syringe, the mixture was stirred in order to prevent sedimentation. The mixed solution was then drawn into a syringe and administered to the patient via enteral tube. After administration, the feeding tube was flushed with $15-30 \mathrm{~mL}$ of water.

\section{Data collection}

Patients who were on any of the six broad-spectrum antibiotics were tracked via Safety Surveillor: a real-time, patient-specific antibiotic reporting system. Pharmacists checked to see if a patient should receive administration of probiotics through real time orders. In the Safety Surveillor data, pharmacy student interns would manually review a patient's electronic medical records for administration of any of the six broad-spectrum antibiotics. Patients who met the criteria were documented and addition of a probiotic was assessed. If there were no probiotics being administered to the patient, the pharmacist assigned to the patient would be alerted by a pharmacy student.

\section{Pharmacist compliance}

A two-step check system of reminding the pharmacist in charge of each patient to order probiotics was utilized. Pharmacy interns would use Safety Surveillor to check for patients on any of the six 
antibiotics, they would subsequently check to see if patients were on probiotics. If not, they would remind the pharmacist assigned to the patient. This was the first step. The next day, pharmacy interns would perform the same check and remind pharmacists again, of which patients should be on probiotics, this was the second step. The second reminder was implemented to assure that all patients on any of the six antibiotics were being given probiotics. After the second step all patients that qualified for probiotics were receiving them.

\section{Results and Discussion}

Analysis of Probiotics administration

In NWH, HO-CDI average rate for 2015 was 8.02 cases per 10,000 patient days. After implementation of probiotics, the current year to date (CYTD) rate of HO-CDI for 2016 was 4.93 and 4.47 for 2017 (Jan-May). The probiotic administration protocol (along with the clean collaborative) appeared to yield a trend towards lower HO-CDI average rates.

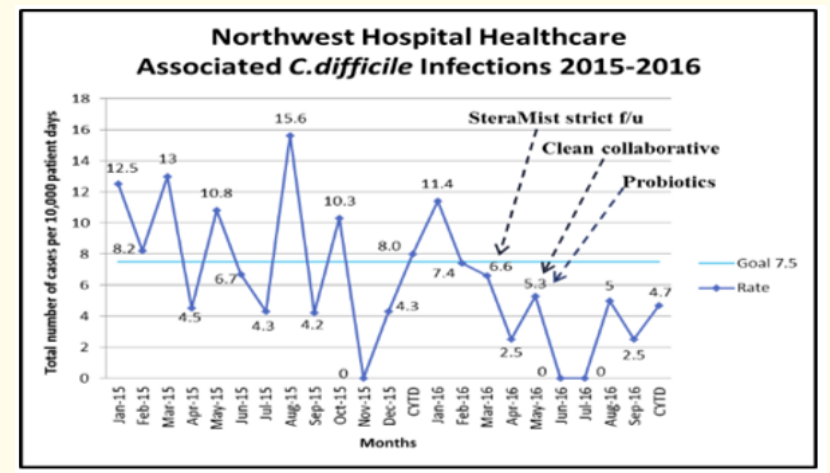

Figure 1: The HO-CDI rates from Jan 2015-Oct 2016 are shown on this graph. The HO-CDI rates for when the probiotics protocol, along with the clean collaborative, began is highlighted on the graph. The goal HO-CDI rate was 7.5 per 10,000 patient days. The graph shows a trend below that goal rate after administration of the probiotics protocol and the clean collaborative.

C. difficile rates per 10,000 patient days from Dec 2015-Apr 2017

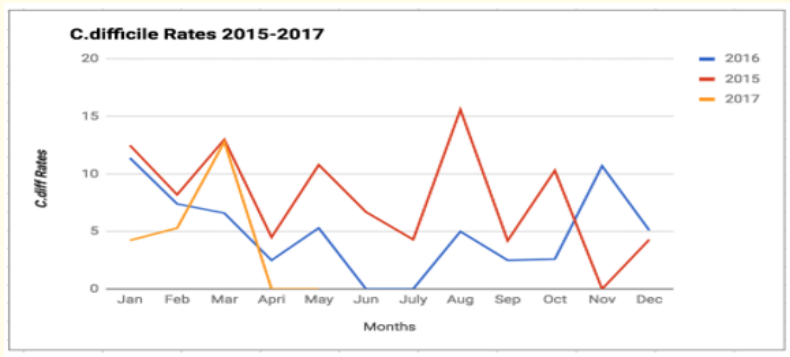

Figure 2: Comparison of $C$. difficile rates per 10,000 patient days from 2015-2017. C. difficile rates from Jan -Dec 2015, Jan Dec 2016, and Jan - May 2017 are displayed. The average rate for: $2015=8.02 ; 2016=4.93 ; 2017=4.47$. The average rate of HO-CDI per 10,000 patient days from the beginning of the study in May 2016 to May 2017 was 4.12 .

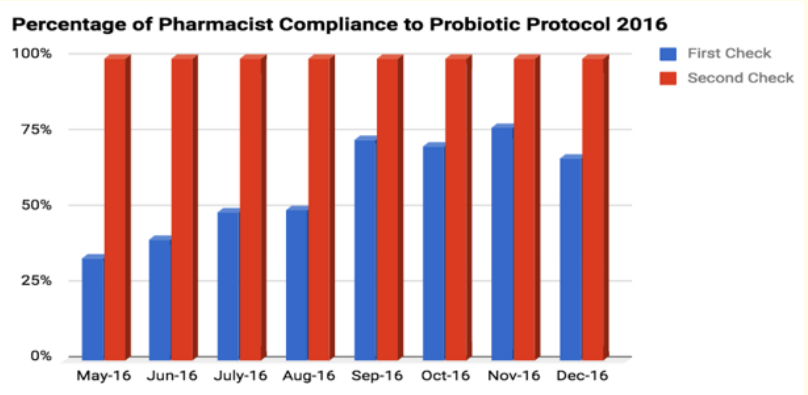

Figure 3: 5/2016-12/2016 average percentage of pharmacist compliance with probiotic protocol for each month for 1st check, and 2nd check. 1st check: Pharmacists check each of their patient's medical records and determine who is qualified for probiotics administration. 2nd check: Pharmacy student interns use SafetySurveilor to alert pharmacists of which patients they may have missed that qualify for probiotics administration. Second check was always $100 \%$ compliance with probiotic administration.

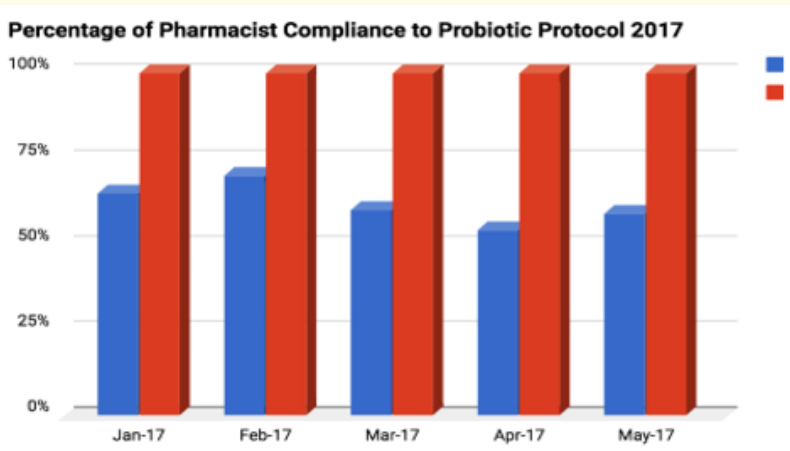

Figure 4: 1/2017-5/2017 average percentage of pharmacist compliance with probiotic protocol for each month for 1st check, and 2 nd check.

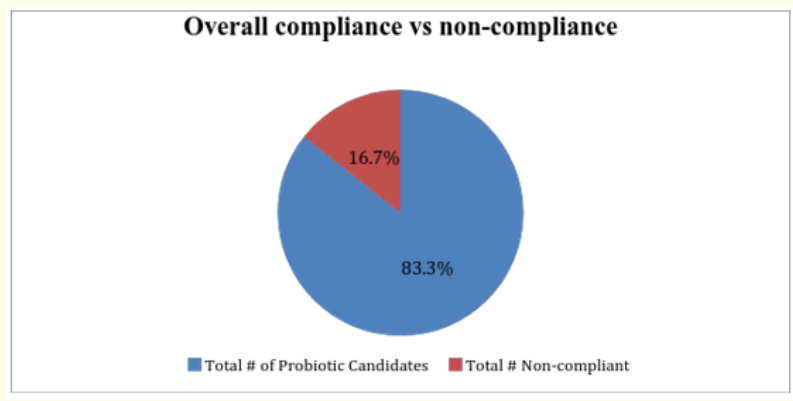

Figure 5: Percentage of patients who were compliant with probiotic administration vs. those that were non-compliant. Total \# of qualified probiotics candidates from from 5/16-5/17 was 7000 , of that 1172 were noncompliant. $14 \%$ of patients who were qualified for probiotics declined administration. 


\section{Conclusion}

The goal of this study was to achieve an HO-CDI rate of 7.5 per 10,000 patient days. This goal was met and exceeded based on the average HO-CDI rate of 4.12 per 10,000 patient days, which was achieved during the duration of the study (May 2016-May 2017). The probiotics administration results further emphasize the importance of early delivery of probiotics during a course of antibiotics. The administration of the probiotics within the first 24 hours of antibiotic initiation, in addition to the Clean Collaborative, was imperative in helping to reduce the chance of CDI. Studies have shown that evidence of probiotics reducing the development of CDI after antibiotic administration is mixed. However, in this program, the data showed a favorable trend toward a decrease in HO-CDI, along with the application of the Clean Collaborative protocol. It is important to note that the patients incurred no harm through the administration of probiotics. There were no incidents of bacteremia or lactobacillus induced sepsis. The two-step protocol for alerting pharmacists to place a probiotic administration order was very effective, especially during the second check. The first check would yield less than $100 \%$ compliance, but once the second check was done, within 24 hours of the first, the compliance rate was always $100 \%$.

A limitation of this study was the inability to include patients that were on parenteral nutrition. This patient population, who otherwise qualified for probiotics, could not benefit from probiotic prophylaxis. These patients may benefit from future studies involving parenterally delivered probiotics. Data continues to be collected on the efficacy of this protocol at NWH. This program was given the 2017 Circle of Honor award for patient safety innovation by the Maryland Safety Center.

\section{Acknowledgements}

The authors would like to thank all of the pharmacy students and pharmacists at Northwest Hospital (Maryland, USA), who participated in the implementation of this program.

\section{Bibliography}

1. Implementation Guide: Guide to preventing Clostridium difficile infections. Association for Professionals in Infection Control and Epidemiology (2017).

2. "Centers for Disease Control and Prevention, CDC. Vital signs: Preventing clostridium difficile infections". MMWR 61 (2012): 157-162.
3. National and State Healthcare Associated Infections Progress Report 2016. Centers for Disease Control and Prevention (2017).

4. Baron S E. Medical Microbiology. 4th edition. Galveston, TX: University of Texas Medical Branch at Galveston (1996).

5. Johnston BC., et al. "Probiotics for the Prevention of Clostridium difficile-Associated Diarrhea: A Systematic Review and Meta-analysis". Annals of International Medicine 157 (2012): 878-888.

6. Marina L Ritchie. "A Meta-Analysis of Probiotic Efficacy for Gastrointestinal Diseases". PLOS (2012).

7. Shen NT., et al. "Timely use of probiotics in hospitalized adults prevents clostridium difficile infection: A systematic review with meta-regression analysis". Gastroenterology 152.8 (2017): 1889,1900.e9.

8. Sazawal S., et al. "Efficacy of probiotics in prevention of acute diarrhoea: A meta-analysis of masked, randomised, placebocontrolled trials". Lancet Infection Disease 6.6 (2006).

\section{Volume 3 Issue 12 December 2019}

(C) All rights are reserved by Cynthia Uche., et al.

Citation: Cynthia Uche., et al. "The Use of Probiotics in the Prevention of $C$. difficile Infection in Admitted Patients Who were Given Broad-Spectrum Antibiotics". Acta Scientific Pharmaceutical Sciences 3.12 (2019): 22-25. 